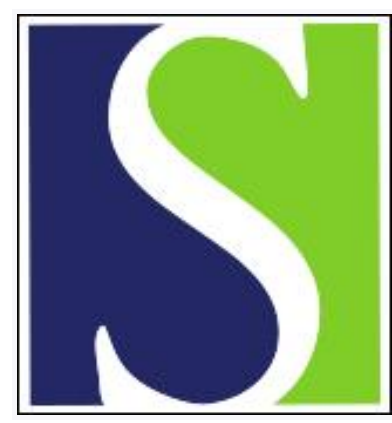

Scand J Work Environ Health 2016;42(3):217-227

https://doi.org/10.5271/sjweh.3560

Issue date: 01 May 2016

Associations between number of consecutive night shifts and impairment of neurobehavioral performance during a subsequent simulated night shift

by Magee M, Sletten TL, Ferguson SA, Grunstein RR, Anderson C, Kennaway DJ, Lockley SW, Rajaratnam SMW

This paper demonstrated increased attentional deficits at the start and end of night shift with a greater amount of night shifts worked. This provide insights into the increased risk of occupational accidents and injuries as the number of consecutive shifts increase and has implications on requirements for fitness for duty assessments prior to the commencement of a night shift.

Key terms: circadian phase; circadian rhythm; neurobehavioral performance; night shift; shift work; shift worker; simulated night shift; sleep; sustained attention

This article in PubMed: www.ncbi.nlm.nih.gov/pubmed/27064758 


\title{
Associations between number of consecutive night shifts and impairment of neurobehavioral performance during a subsequent simulated night shift
}

by Michelle Magee PhD, ${ }^{1,2}$ Tracey L Sletten, PhD, ${ }^{1,2}$ Sally A Ferguson, PhD, ${ }^{3}$ Ronald R Grunstein, MD, 2, 4 Clare Anderson, PhD, ${ }^{1,2,6}$ David J Kennaway, PhD, ${ }^{5}$ Steven W Lockley, PhD, ${ }^{1,2,6}$ Shantha MW Rajaratnam, $P h D^{1,2,6}$

\begin{abstract}
Magee M, Sletten TL, Ferguson SA, Grunstein RR, Anderson C, Kennaway DJ, Lockley SW, Rajaratnam SMW. Associations between number of consecutive night shifts and impairment of neurobehavioral performance during a subsequent simulated night shift. Scand J Work Environ Health. 2016;42(3):217-227. doi:10.5271/sjweh.3560
\end{abstract}

\begin{abstract}
Objective This study aimed to investigate sleep and circadian phase in the relationships between neurobehavioral performance and the number of consecutive shifts worked.

Methods Thirty-four shift workers [20 men, mean age 31.8 (SD 10.9) years] worked 2-7 consecutive night shifts immediately prior to a laboratory-based, simulated night shift. For 7 days prior, participants worked their usual shift sequence, and sleep was assessed with logs and actigraphy. Participants completed a 10-minute auditory psychomotor vigilance task (PVT) at the start ( 21:00 hours) and end ( 07:00 hours) of the simulated night shift. Mean reaction times (RT), number of lapses and RT distribution was compared between those who worked 2-3 consecutive night shifts versus those who worked 4-7 shifts.
\end{abstract}

Results Following 4-7 shifts, night shift workers had significantly longer mean RT at the start and end of shift, compared to those who worked 2-3 shifts. The slowest and fastest $10 \%$ RT were significantly slower at the start, but not end, of shift among participants who worked 4-7 nights. Those working 4-7 nights also demonstrated a broader RT distribution at the start and end of shift and had significantly slower RT based on cumulative distribution analysis $\left(5^{\text {th }}, 25^{\text {th }}, 50^{\text {th }}, 75^{\text {th }}\right.$ percentiles at the start of shift; $75^{\text {th }}$ percentile at the end of shift). No group differences in sleep parameters were found for 7 days and 24 hours prior to the simulated night shift.

Conclusion A greater number of consecutive night shifts has a negative impact on neurobehavioral performance, likely due to cognitive slowing.

Key terms circadian rhythm; circadian phase; shift work; shift worker; sleep; sustained attention.

The 24-hour society has increased the demand for both longer shift durations and night shift work. It is estimated that $\sim 16 \%$ of Australians (1) and $20 \%$ of Americans (2) are engaged in shift work, defined as any work conducted outside of normal working hours. Approximately $60 \%$ of night shift workers report sleep difficulties (3), likely due to the misalignment between the endogenous circadian pacemaker and the individual's work-imposed sleepwake schedule $(4,5)$. This misalignment of sleep from the circadian pacemaker, and the ensuing accumulation of sleep debt, gives rise to increased sleepiness, deficits in neurobehavioral function (6) and adverse safety outcomes $(7,8)$. In simulated night shift work studies, a decline in sustained attention (9), selective attention (10) and cognitive throughput (9) on shift has been reported.

Theoretically, if the number of consecutive night shifts is sufficient, it is possible to adapt the circadian clock to a night shift schedule $(11,12)$ as the circadian

1 Monash Institute of Cognitive and Clinical Neurosciences, School of Psychological Sciences and, Monash University, Melbourne, Australia.

2 NeuroSleep: Centre for Research Excellence for Translational Sleep and Circadian Neurobiology, Sydney, Australia.

3 Central Queensland University, Appleton Institute, Adelaide, Australia.

4 Woolcock Institute of Medical Research, University of Sydney, and Department of Respiratory \& Sleep Medicine, Royal Prince Alfred Hospital, Sydney, Australia.

5 Robinson Research Institute, School of Medicine, Discipline of Obstetrics and Gynaecology, University of Adelaide, Adelaide, Australia.

6 Division of Sleep and Circadian Disorders, Department of Medicine and Department of Neurology, Brigham and Women's Hospital and Division of Sleep Medicine, Harvard Medical School, Boston, Massachusetts, USA.

Correspondence to: Shantha M.W. Rajaratnam, School of Psychological Sciences and Monash Institute of Cognitive and Clinical Neurosciences, Monash University, Building 17, Wellington Road, Clayton VIC, 3800, Australia. [Email: shantha.rajaratnam@monash.edu] 
pacemaker gradually re-entrains to the new light-dark and sleep-wake cycles, which in turn should result in improved alertness and neurobehavioral outcomes during the night (13). The rate of shift is slow, however, with only $1-2$ hours per day of readaptation $(11,12)$. Under most circumstances, conflicting light-dark exposure, coupled with the tendency to revert to day-active schedules on days off from work mean that, in practice complete circadian adaptation is relatively uncommon. A meta-analysis of six studies ( $\mathrm{N}=76$ individuals) using the melatonin rhythm as a marker of circadian phase adaptation among permanent night shift workers reported that $<3 \%$ showed complete circadian adaptation to night shift work, and $21 \%$ showed partial adaptation (4). A study of miners working seven 12-hour day and night shifts provided no evidence of circadian adaption of melatonin rhythmicity and progressive impairment of reaction times and vigilance across the week (14). In contrast, undertaking seven consecutive simulated night shifts, demonstrated decreased performance over seven nights coinciding with partial circadian adaptation and adequate day sleep opportunity (13). It is unclear, therefore, the extent to which a greater number of consecutive night shifts facilitates adaptation and subsequent improved alertness outcomes among night shift workers.

Analysis of workplace accident reports indicate that the risk of accidents and injuries increases with the number of consecutive night shifts worked (15). For example, Folkard and Lombardi (16) reported that the relative risk of incident was $\sim 6 \%$ higher on the second consecutive night worked (compared to the first), $17 \%$ higher on the third night, and $36 \%$ higher on the fourth consecutive night worked. Based broadly on this and other work, a number of industries limit the number of consecutive night shifts that can be worked. For example, the American Institute of Medicine (IOM) now recommends that residents should work $\leq 4$ consecutive night shifts with each series followed by $\geq 48$ hours rostered off.

Given the limited empirical data to support the claim that increasing the number consecutive night shifts may have deleterious effects on alertness and neurobehavioral performance, this study examined neurobehavioral performance impairments among night shift workers during a laboratory-based, simulated night shift, immediately after they had worked either $4-7$ or $2-3$ consecutive night shifts. Using both probability and cumulative reaction time (RT) distribution analysis, we examined the specific nature of these neurobehavioral impairments, specifically inattention versus sensory motor speed, information processing, and cognitive slowing $(10,17,18)$. We also aimed to examine the influences of sleep and circadian phase in the relationships between neurobehavioral performance and the number of consecutive shifts worked.

\section{Methods}

\section{Ethical approval}

The Human Research Ethics Committees of both Monash University and the University of Sydney approved the study protocol. All participants provided written informed consent and were reimbursed for study-related expenses. The data presented here are taken from a larger, multi-center randomized controlled trial testing the efficacy of a novel light exposure intervention to improve alertness and neurobehavioral performance among night shift workers. Only data from the standard (4000 K) light condition are presented here. We have previously reported results from this study showing the association between circadian rhythm phase and neurobehavioral impairment (19).

\section{Participant recruitment}

Thirty-four healthy shift workers (20 males, 14 females), aged 18-64 years (mean 31.8, SD 10.9, years) with a normal body mass index (BMI) (mean 24.5, SD 3.6, kg/ $\mathrm{m}^{2}$ ) were recruited from a range of occupational settings (see table 1): professionals $(\mathrm{N}=13)$, managers $(\mathrm{N}=3)$, medical technicians $(\mathrm{N}=2)$, community and personal service workers $(\mathrm{N}=8)$, machinery operators and drivers $(\mathrm{N}=4)$, technicians and trade workers $(\mathrm{N}=2)$ and unknown occupation ( $\mathrm{N}=2)$. Night shift work was defined as $\geq 6$ hours worked between 22:00-8:00 hours with a maximum shift length of 12 hours. Participants were required to work at least 2 but no more than 7 consecutive night shifts prior to their laboratory visit, as confirmed by work diaries. For the purpose of this study "night shift worker" was defined accordingly.. Of the participants, $77.8 \%$ engaged in consecutive shift work in the seven days prior to the simulated night shift, while $22.2 \%$ worked additional shifts in the seven days days prior. Exclusion criteria were history/presence of major medical, neurological, visual, auditory, sleep or psychiatric disorder; BMI $>30$ $\mathrm{kg} / \mathrm{m} 2$; high risk of obstructive sleep apnea according to the Berlin Questionnaire (20); travel $>2$ time zones in the three months prior to the study; excessive consumption of caffeine ( $>500 \mathrm{mg} /$ day); nicotine consumption equivalent to $>5$ cigarettes/day; alcohol consumption $>14$ units/ week; or illicit drug use.

\section{Pre-laboratory assessment}

Subjective measures of general health and sleep (sleep quality, sleepiness, sleep hygiene and morning/evening preference) were assessed at an initial screening visit. The Short Form-36 (SF-36) Health Survey was administered to measure quality of life (21), where the global score is used as an indicator of general health. The Pittsburgh 
Table 1. Participant demographics based on number of nights worked consecutively. [ESS=Epworth sleepiness scale; $P S Q I=P i t t s b u r g h$ sleep quality index; $S D=$ standard deviation; SF$36=$ short form -36 health survey; $M E Q=$ morningness-eveningness questionnaire.]

\begin{tabular}{|c|c|c|c|c|c|c|c|c|c|}
\hline & \multicolumn{4}{|c|}{$2-3$ nights } & \multicolumn{4}{|c|}{ 4-7 nights } & \multirow[t]{2}{*}{ P-value } \\
\hline & $\mathrm{N}$ & $\%$ & Mean & $\mathrm{SD}$ & $\mathrm{N}$ & $\%$ & Mean & $\mathrm{SD}$ & \\
\hline Demographics & 18 & & & & 16 & & & & \\
\hline Male & 11 & & & & 9 & & & & 0.77 \\
\hline Female & 7 & & & & 7 & & & & \\
\hline Age (years) & & & 27.5 & 7.2 & & & 36.7 & 12.4 & 0.02 \\
\hline \multicolumn{10}{|l|}{ Occupation } \\
\hline Professionals & 7 & 38.9 & & & 9 & 56.3 & & & \\
\hline $\begin{array}{l}\text { Community \& } \\
\text { personal ser- } \\
\text { vice workers }\end{array}$ & 7 & 38.9 & & & 1 & 6.3 & & & \\
\hline Technicians & 2 & 11.1 & & & 2 & 12.5 & & & \\
\hline $\begin{array}{l}\text { Machinery } \\
\text { operators \& } \\
\text { drivers }\end{array}$ & 1 & 5.6 & & & 3 & 18.75 & & & \\
\hline Unknown & 1 & 5.6 & & & 1 & 6.25 & & & \\
\hline \multicolumn{10}{|l|}{ General health } \\
\hline SF-36 & & & 99.6 & 6.5 & & & 100.7 & 3.5 & 0.41 \\
\hline BMI $\left(\mathrm{kg} / \mathrm{m}^{2}\right)$ & & & 24.5 & 3.7 & & & 24.8 & 3.6 & 0.70 \\
\hline \multicolumn{10}{|c|}{ Sleep-wake measures } \\
\hline $\begin{array}{l}\text { Sleep hygiene } \\
\text { index }\end{array}$ & & & 33.0 & 4.5 & & & 33.3 & 7.0 & 0.99 \\
\hline MEQ score & & & 51.6 & 8.3 & & & 51.8 & 12.1 & 0.84 \\
\hline ESS & & & 8.4 & 4.8 & & & 7.8 & 4.4 & 0.69 \\
\hline$<10$ & 13 & 70.6 & & & 10 & 62.5 & & & 0.55 \\
\hline$\geq 10$ & 5 & 29.4 & & & 6 & 37.5 & & & \\
\hline PSQI score & & & 6.4 & 2.6 & & & 6.6 & 2.7 & 0.98 \\
\hline$<5$ & 6 & 33.3 & & & 4 & 25.0 & & & 0.93 \\
\hline$\geq 5$ & 12 & 66.7 & & & 12 & 75.0 & & & \\
\hline \multicolumn{10}{|c|}{$\begin{array}{l}\text { Work schedule } \\
\text { history } 7 \text { days prior }\end{array}$} \\
\hline $\begin{array}{l}\text { Consecutive } \\
\text { nights worked } \\
\text { (number) }\end{array}$ & & & 2.2 & 0.4 & & & 5.1 & 1.3 & $<0.001$ \\
\hline $\begin{array}{l}\text { Hours worked } \\
\text { (hours) }\end{array}$ & & & 19.3 & 5.8 & & & 47.4 & 11.6 & $<0.001$ \\
\hline $\begin{array}{l}\text { Duration of } \\
\text { shift (hours) }\end{array}$ & & & 8.6 & 1.8 & & & 9.4 & 1.2 & 0.17 \\
\hline
\end{tabular}

Sleep Quality Index (PSQI) was administered to determine sleep quality, where a global score $>5$ was indicative of poor sleep quality (22). Daytime sleepiness was assessed with the Epworth Sleepiness Scale (ESS) (23), whereby ESS scores $\geq 10$ indicated excessive daytime sleepiness (23). Sleep hygiene behaviors were assessed using the Sleep Hygiene Index (24), with higher scores indicating maladaptive sleep hygiene practices. Morning/ evening preference was determined through use of the Morningness-Eveningness Questionnaire (MEQ) (25).

Participants completed a sleep diary and wore a wrist actigraphy device (Actiwatch-64 or Actiwatch-L; Respironics, Bend, OR, USA) to assess rest-activity patterns (in 1 minute epochs at medium sensitivity; 40 activity counts per epoch) for seven days prior to the laboratory visit. Sleep diary data were used to estimate bed and wake time, time in bed, and daily number of awakenings for main sleep episodes and naps. Bed and wake times as reported in sleep diaries were used to determine the actigraphy analysis interval for each sleep episode (Actiware 5 software, Respironics Inc, Bend, OR, USA). The following variables were computed for the main sleep episodes: time in bed, total sleep time, sleep efficiency, and sleep onset latency.

Participants were also instructed to document their work schedules for seven days prior to the laboratory visit. Participants reported hours worked, duration of breaks, pre- and post-work sleepiness ratings ( $1=$ extremely alert, $10=$ very sleepy, fighting sleep, an effort to stay awake) and shift workload ratings (1=very light, $5=$ very heavy).

\section{Simulated night shift protocol}

After completing a series of night shifts in their usual workplace, participants attended the laboratory from 17:30-09:00 hours, with a simulated night shift from 21:00-07:00 hours. Prior to the laboratory visit, participants were instructed to attempt sleep for $\geq 6$ hours after completing their last night shift. Participants were instructed to abstain from nicotine use and caffeine consumption for 12 hours prior to the simulated night shift, and abstain from alcohol for 24 hours prior to the shift. On arrival at the laboratory, participants were screened for illicit drug use via urine toxicology. Participants were required to remain awake in standard lighting conditions (4000 K; 100 lux at eye level, $137 \mathrm{~cm}$ from floor, vertical plane) and were monitored continuously by trained staff. Between testing, participants remained seated, however, they were permitted to complete quiet tasks such as reading, doing puzzles and watching movies. They were provided with calorie-controlled meals (20:20 hours, 06:20 hours), snacks (22:20 hours, 01:20 hours) and water was provided ad libitum. Throughout the protocol, toilet breaks were scheduled every two hours.

\section{Neurobehavioral performance}

The auditory Psychomotor Vigilance Task (PVT, 10 minutes, inter-stimulus interval 1-9 seconds), a wellestablished measure of sustained attention $(26,27)$, was administered at 21:00 hours (at the start of the simulated shift) and 07:00 hours (end of simulated shift) using E-Prime 2.0 software (Psychology Software Tools, Inc., Sharpsburg, PA, USA). The auditory PVT was chosen as it is less variable than visual PVT (28). RT $<100 \mathrm{~ms}$ (29) and $>10,000 \mathrm{~ms}$ (17) were excluded from the analysis.

Based on previous sleep loss studies (26), the following variables were extracted for each PVT session: mean of slowest $10 \%$ responses, mean of fastest $10 \%$ responses, mean RT and number of lapses (RT $>500 \mathrm{~ms}$ ). To normalize data, mean RT, mean slowest $10 \%$ responses and mean fastest $10 \%$ responses were expressed as $\log (\mathrm{RT})$. Lapses (x) were normalized and 
expressed as $\sqrt{ } \mathrm{x}+\sqrt{ }(\mathrm{x}+1)$. Two participants did not complete the PVT at the end of the shift. For analysis purposes, the calculated average $\log (\mathrm{RT})$ and normalized average number of lapses for all participants at the end of shift were substituted for these two participants.

Cumulative and probability plots of whole RT distribution were computed for a more extensive characterisation of performance deficits not necessarily captured by investigating typical PVT summary statistics alone $(10,18,30)$. Group differences in neurobehavioral performance were examined by computing cumulative and probability distribution plots. For each participant, each response was normalized by converting to $\log$ (RT) and sorted in descending order (fastest to slowest). The 5-95 th percentiles ( $5 \%$ increments) of RT data were calculated for each participant and group differences were examined using analysis of covariance (ANCOVA) at the $5^{\text {th }}, 25^{\text {th }}$, $75^{\text {th }}$ and $95^{\text {th }}$ percentiles controlling for age. To examine the distribution of RT responses, a 4-parameter Weibull distribution curve was fitted to the normalized percentile data for each participant at the start and end of the simulated shift, The 4-parameter Weibull curve has been previously shown to have good fit to $\log$ (RT) data that is nonnormally distributed $(10,17,18,30)$ (ie, a Gaussian shape with a long tail), and provides four parameters which are thought to reflect varying attentional processes: (i) shift - displacement of the distribution reflecting sensory/ motor speed; (ii) scale - distribution spread representing information processing speed; (iii) shape - skewness of the distribution; and (iv) midpoint $\left(\mathrm{X}_{0}\right)$ the $50^{\text {th }}$ percentile - median $(10,18)$. Weibull parameters that did not predict the $\log (\mathrm{RT})$ distribution $(\mathrm{P}>0.05)$ were excluded from analysis ( $\mathrm{N}=18$ of $272,6.61 \%$ excluded from data analysis). Group differences in Weibull parameters shift (A), scale (B) shape (C), and midpoint $\left(\mathrm{X}_{0}\right)$ were examined for the start (21:00 hours) and end (07:00 hours).

\section{Urinary 6-sulphatoxymelatonin}

Forty-eight hours immediately prior to the simulated night shift, a subset of participants who worked 2-3 $(\mathrm{N}=10)$ or 4-7 ( $\mathrm{N}=8)$ nights consecutively collected urine samples at approximately 4-hour intervals (8-hour intervals during sleep episodes) for measurement of the urinary metabolite of melatonin, 6-sulphatoxymelatonin (aMT6s). After each collection, participants recorded the volume of the sample and the time of urine collection. A 5-ml urine aliquot was frozen $\left(-20^{\circ} \mathrm{C}\right)$ and subsequently analysed by radioimmunoassay (31) at the Adelaide Research Assay Facility, University of Adelaide, using reagents purchased from Stockgrand Ltd, University of Surrey Guildford, UK. The aMT6s intra-assay coefficient of variation (CV) was $7.2 \%$ and the inter-assay $\mathrm{CV}$ were $23 \%, 7 \%$ and $11 \%$ at $3.6 \mathrm{ng} / \mathrm{ml}, 15.6 \mathrm{ng} / \mathrm{ml}$ and $29.6 \mathrm{ng} / \mathrm{ml}$, respectively. The minimum detectable concentration was $0.5 \mathrm{ng} / \mathrm{ml}$.

\section{Data analysis}

Statistical analyses were conducted using SPSS version 21.0 (SPSS Inc, Chicago, IL, USA). For participant demographics, between group differences (number of nights worked, 2-3 versus 4-7 nights) were examined using independent student's t-tests and Chi-square analysis. Where data were not normally distributed, Mann Whitney U test was used. Group differences in sleep parameters (both 24 hours and 7 days prior to the simulated night shift) and PVT performance metrics were assessed using analysis of co-variance (ANCOVA) controlling for age. When normality was violated, data were transformed as specified in figures and tables. PVT data were fitted with the Weibull distribution curve using Sigmaplot 2008 (Systat Software Inc, San Jose, CA, USA) and Weibull distribution parameters were also examined at both the start and end of the simulated night using ANCOVA, controlling for age based on previous reports of age-related changes in response to sleep loss (32). Cosinor analysis of aMT6s values was used to determine acrophase (peak) time of the aMT6s rhythm (33). An alpha of $<0.05$ was accepted as significant.

\section{Results}

\section{Data retention}

Of the 34 participants, $4(11.7 \%)$ did not have actigraphy data available for the 24 hours prior to the simulated night shift. Furthermore, 5 (14.7\%) participants did not complete the seven days of actigraphy and/or sleep diary entries prior to the simulated night shift.

\section{Participant demographics}

Demographics, general health, sleep wake measures and night shift work history are reported in table 1. Participants working 4-7 nights were significantly older [mean $36.7(\mathrm{SD} 12.4)$ years; $\mathrm{t}(23.5)=-2.6, \mathrm{P}=0.02]$ in comparison to participants who worked 2-3 nights [mean 27.5 (SD 7.2) years]. As expected, the total number of hours worked in the seven days prior was significantly higher among participants who worked 4-7 nights [mean 47.4 (SD 11.6) hours; $\mathrm{t}(21.1)=-8.9, \mathrm{P}<0.001]$ compared to those who worked 2-3 nights [mean 19.1 (SD 5.6) hours]. While the total number of shifts and hours worked differed between groups, the average duration of shifts did not differ.

\section{Sleep-wake measures prior to the simulated night shift}

Actigraphic time in bed, total sleep time, sleep effi- 
ciency, and sleep onset latency in the week before the laboratory visit did not differ between groups (table 2). This was the case for both seven days and 24 hours prior to the simulated night shift. There was a trend for a longer sleep onset latency among participants who worked $4-7$ nights $[F(1,28)=2.37, \mathrm{P}=0.08]$.

\section{Circadian phase assessment prior to the simulated night shift}

In a subset of night shift workers, cosinor analysis of urinary aMT6s rhythm were used to determine acrophase time (peak of aMT6s), as previously described (34), among participants who worked 2-3 nights consecutively $(\mathrm{N}=10)$ compared to those who worked $4-7$ nights $(\mathrm{N}=8)$ (table 2). We found no significant differences in the timing of the aMT6s rhythm acrophase among those who worked 2-3 nights [mean 04:53 (SD 2:34) hours] and those who had worked 4-7 nights [mean 06:39 (SD 04:13) hours; $\mathrm{t}(16)=-1.05, \mathrm{P}=0.31]$.

\section{Effect of consecutive night shifts on PVT metrics}

Compared to 2-3 nights worked, participants who worked 4-7 nights had a significantly slower mean RT $[F(1,31)=$ 7.34, $\mathrm{P}=0.01]$, fastest $10 \% \mathrm{RT}[F(1,31)=5.88, \mathrm{P}=0.02]$ (figure 1C) and slowest $10 \% \mathrm{RT}[F(1,31)=5.41, \mathrm{P}=0.03]$ (figure 1D) at the start of the simulated night shift. There was no difference between groups in the number of lapses at the beginning of shift, however a trend to greater number of lapses were seen the participants who worked 4-7 consecutive nights $[F(1,31)=3.68, \mathrm{P}=0.06]$. At the end of the simulated night shift, participants who worked 4-7 consecutive nights also demonstrated longer mRT $[F(1,31)=4.15, \mathrm{P}=0.05]$ (figure $1 \mathrm{~A}$ ), a trend to longer slowest $10 \%$ RT $[F(1,31)=3.61, \mathrm{P}=0.07]$ and a trend to greatest lapses at end of shift $[F(1,31)=3.65, \mathrm{P}=0.07]$ (figure 1B). There were no group differences in $10 \%$ fastest RT $[F(1,31)=5.88, \mathrm{P}=0.55]$ at the end of shift. No interaction effects were demonstrated between group differences in performance and time of shift (start and end) (analysis not shown).

\section{Consecutive night shifts effect on whole RT distribution}

At the start and end of shift, there were no differences in shift (A), scale (B), or shape (C) Weibull parameters between groups (table 3 ). There was, however, a significant difference in midpoint $\mathrm{RT}\left(\mathrm{X}_{0}\right)$, where participants who worked 4-7 nights demonstrated a slower median RT at the start of shift compared to those who worked $2-3$ consecutive nights $[F(1,31)=9.13$, $\mathrm{P}=0.01$ ], suggestive of cognitive slowing. No changes in midpoint RT were noted at the end of shift between groups. No interaction effects were demonstrated in any
Table 2. Participant sleep/wake characteristics based on consecutive nights worked. Analysis of variance performed controlling for age. [aMT6s=6-sulphatoxymelatonin; SD=standard deviation; SE=sleep efficiency; SOL=sleep onset latency; TIB=time in bed; TST=total sleep time.]

\begin{tabular}{|c|c|c|c|c|c|c|c|}
\hline & \multicolumn{3}{|c|}{$2-3$ nights $^{\text {a }}$} & \multicolumn{3}{|c|}{$4-7$ nights $^{b}$} & \multirow[t]{2}{*}{$P$ value } \\
\hline & $\mathrm{N}$ & Mean & SD & $\mathrm{N}$ & Mean & SD & \\
\hline $\begin{array}{l}7 \text { days prior to } \\
\text { simulated night shift }\end{array}$ & 15 & & & 14 & & & \\
\hline Total TIB (hours) & & 53.5 & 8.8 & & 48.0 & 10.9 & 0.44 \\
\hline Mean TIB (hours) & & 6.8 & 1.6 & & 6.2 & 1.5 & 0.55 \\
\hline Total TST (hours) & & 48.7 & 8.3 & & 43.3 & 11.1 & 0.32 \\
\hline Mean TST (hours) & & 6.3 & 1.4 & & 5.6 & 1.4 & 0.34 \\
\hline SE $(\%)$ & & 77.1 & 7.9 & & 70.3 & 18.4 & 0.14 \\
\hline SOL (minutes) & & 14.2 & 11.9 & & 22.1 & 22.7 & 0.08 \\
\hline $\begin{array}{l}24 \text { hours prior to } \\
\text { simulated night shift }\end{array}$ & 15 & & & 16 & & & \\
\hline TIB (hours) & & 6.9 & 1.4 & & 5.9 & 1.4 & 0.20 \\
\hline TST (hours) & & 6.3 & 1.5 & & 5 & 1.4 & 0.2 \\
\hline SE (\%) & & 79.4 & 10.3 & & 79.8 & 11.8 & 0.66 \\
\hline SOL (minutes) & & 16.2 & 21.2 & & 16.3 & 3.4 & 0.78 \\
\hline $\begin{array}{l}\text { Wake prior to night } \\
\text { shift (hours) }\end{array}$ & & 6.0 & 1.7 & & 6.2 & 1.7 & 0.55 \\
\hline $\begin{array}{l}\text { Circadian phase } \\
\text { assessment }\end{array}$ & 10 & & & 8 & & & \\
\hline $\begin{array}{l}\text { aMT6s acrophase } \\
\text { (hours) }\end{array}$ & & $4: 53$ & $2: 34$ & & 6:39 & $4: 13$ & 0.31 \\
\hline
\end{tabular}

a Range for circadian phase assessment: 00:48-08:47 hours.

${ }^{b}$ Range for circadian phase assessment: 23:17-12:16 hours.

Table 3. Weibull analysis parameters at the start and end of a simulated night shift separated by the number of consecutive nights worked prior. Analysis of covariance performed controlling for age. $[A=$ shift (sensory motor speed); $B=$ scale (information processing speed); $\mathrm{C}=$ shape (measure of skew of the distribution); $\mathrm{X}_{0}=$ median $50^{\text {th }}$ percentile; $\mathrm{SD}=$ standard deviation.]

\begin{tabular}{|c|c|c|c|c|c|c|c|c|}
\hline & \multicolumn{4}{|c|}{$2-3$ nights } & \multicolumn{4}{|c|}{$4-7$ nights } \\
\hline & \multicolumn{2}{|c|}{ Start } & \multicolumn{2}{|c|}{ End } & \multicolumn{2}{|c|}{ Start } & \multicolumn{2}{|c|}{ End } \\
\hline & Mean & SD & Mean & $\mathrm{SD}$ & Mean & $\mathrm{SD}$ & Mean & SD \\
\hline A & 98.0 & 4.2 & 105.2 & 24.2 & 102.7 & 12.5 & 95.77 & 4.07 \\
\hline B & 0.16 & 0.11 & 0.23 & 0.09 & 0.16 & 0.06 & 0.19 & 0.05 \\
\hline C & 2.33 & 0.93 & 2.68 & 1.18 & 2.56 & 1.36 & 2.35 & 1.12 \\
\hline $\mathrm{X}_{0}$ & 2.41 & 0.05 & 2.49 & 0.07 & $2.46^{\mathrm{a}}$ & 0.05 & 2.50 & 0.06 \\
\hline
\end{tabular}

a $P<0.001$ 2-3 nights 21:00 hours versus $4-7$ nights 21:00 hours.

of the Weibull parameters $(\mathrm{P}>0.05)$.

At the start of shift significantly slower RT were observed at the $25^{\text {th }}[F(1,31)=9.06, \mathrm{P}=0.005], 50^{\text {th }}[F$ $(1,31)=9.12, \mathrm{P}=0.005], 75^{\text {th }}[F(1,31)=6.61, \mathrm{P}=0.02]$ and $95^{\text {th }}$ percentile $[F(1,31)=4.25, \mathrm{P}=0.05]$ among participants who worked 4-7 nights (figure 2A). A trend to slower RT was also noted at the $5^{\text {th }}$ percentile $[F(1,31)$ $=3.84, \mathrm{P}=0.059]$ among participants who worked 4-7 nights. At the end of shift, slower RT were observed at the $75^{\text {th }}$ percentile $[F(1,33)=4.30, \mathrm{P}=0.05]$ and a trend to slower RT at the $95^{\text {th }}$ percentile $[F(1,33)=3.78$, $\mathrm{P}=0.06]$ in participants who worked 4-7 nights (figure $2 \mathrm{C})$. No group differences were observed at the $5^{\text {th }}[F$ $(1,31)=0.41, \mathrm{P}=0.527], 25^{\text {th }}[F(1,31)=1.23, \mathrm{P}=0.275]$ 


\section{A. Mean RT}

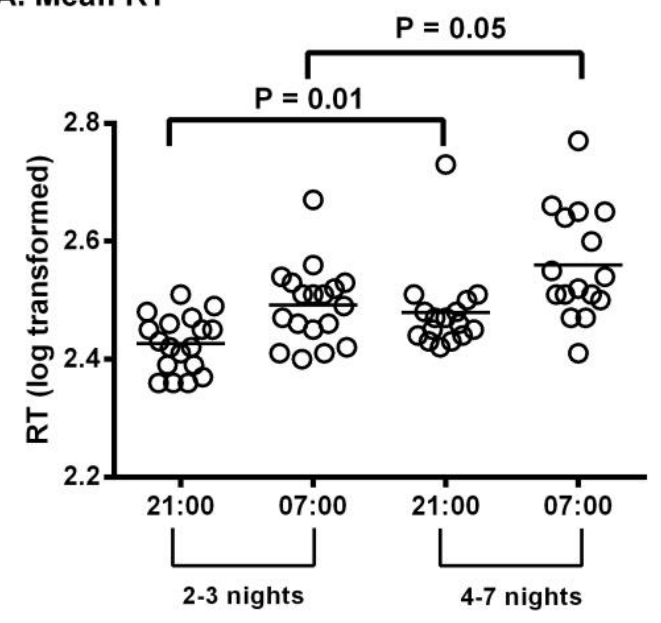

C. Fastest $10 \%$ RT

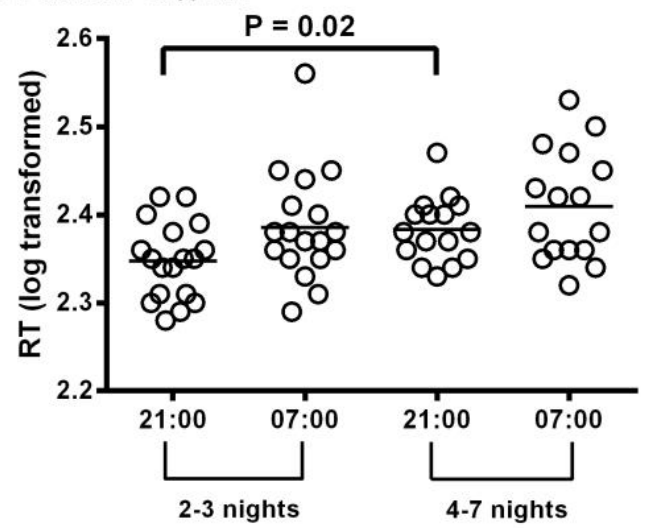

B. Number of lapses

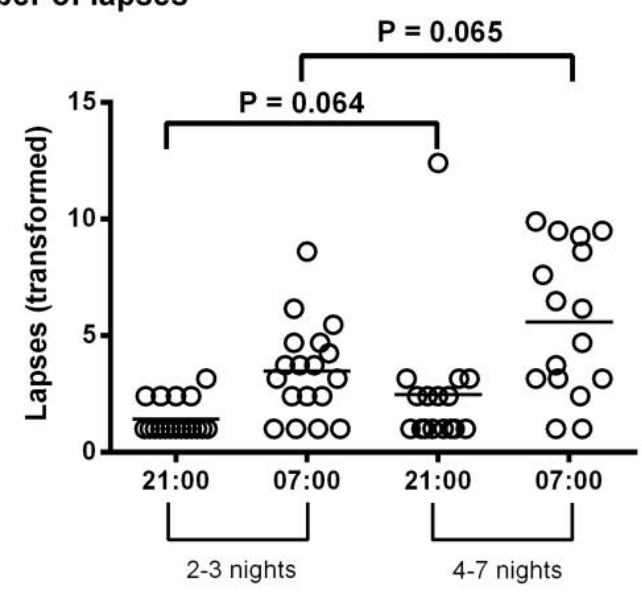

D. Slowest $10 \%$ RT

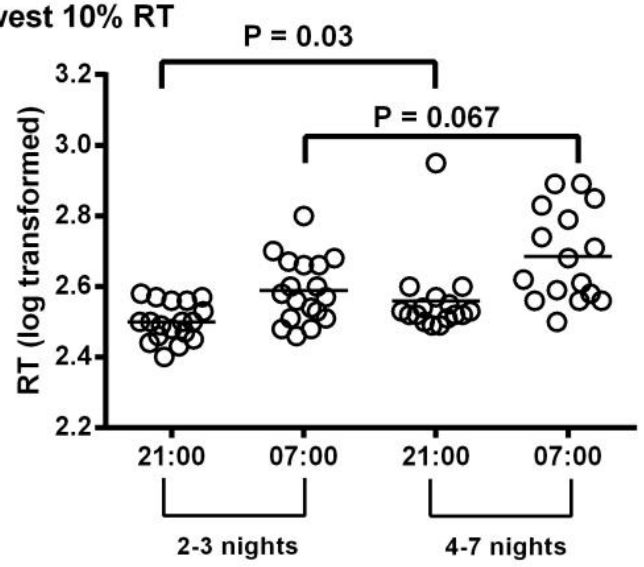

Figure 1. Neurobehavioural performance (PVT metrics) among night shift workers who work either 2-3 or 4-7 consecutive nights, was measured during a simulated night shift at both the start $(21: 00)$ and end (07:00) of the shift. (A) Represents mRT; (B) Number of lapses (Tukey transformed $[\sqrt{ }(x)+\sqrt{ }(x+1)]) ;(C) 10 \%$ slowest $R T$; and (D) $10 \%$ fastest $R T$. All values reported are transformed variables. All greater changes indicate increased neurobehavioural performance impairment.

and $50^{\text {th }}[F(1,31)=2.66, \mathrm{P}=0.113]$ percentiles.

While the RT distribution shape in both groups were similar (normal distribution of RT with a long tail), participants who worked 4-7 nights demonstrated a rightward shift in RT distribution at both the start (figure 2B) and end of shift (figure 2D).

\section{Discussion}

This study used a combination of field and in-laboratory procedures to examine, in a sample of night shift workers, the impact of prior consecutive night shifts on neurobehavioral performance at the start and end of a subsequent simulated night shift. Individuals who worked 4-7 consecutive nights exhibited a greater degree of neurobehavioral impairment at the start and end of the shift compared to those who had worked 2-3 nights. Specifically, those who had worked 4-7 nights had longer mean, slowest $10 \%$ and fastest $10 \%$ RT; longer RT at the $25^{\text {th }}, 50^{\text {th }}, 75^{\text {th }}$ and $95^{\text {th }}$ percentiles at the start of shift; and longer mean RT and RT at the $75^{\text {th }}$ percentile at the end of shift. Together, these results are indicative of cognitive slowing $(17,18)$, particularly at the start of a night shift among those who had previously worked 4-7 consecutive night shifts. Sleep parameters during the seven days and 24 hours prior to the simulated night shift did not differ between groups.

Although previous studies have shown associations between the number of consecutive night shifts and the magnitude of performance decrements under both controlled laboratory conditions $(35,36)$ and in operational settings $(37,38)$, to our knowledge, no other study has observed PVT performance decrements at the start of shift as a function of number of consecutive night shifts in a sample of shift workers. In a study investigating permanent night shifts workers, no changes 
A

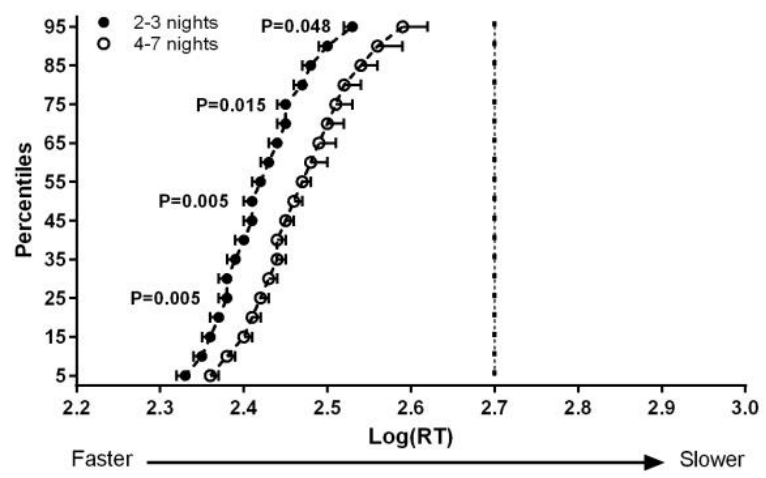

C

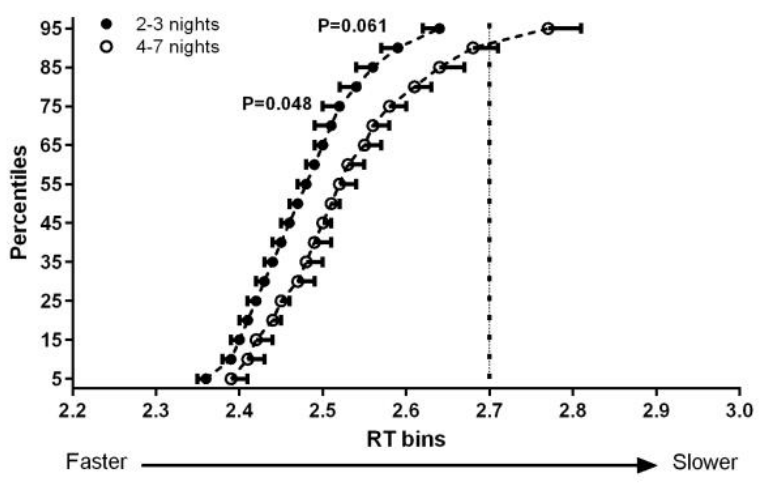

B

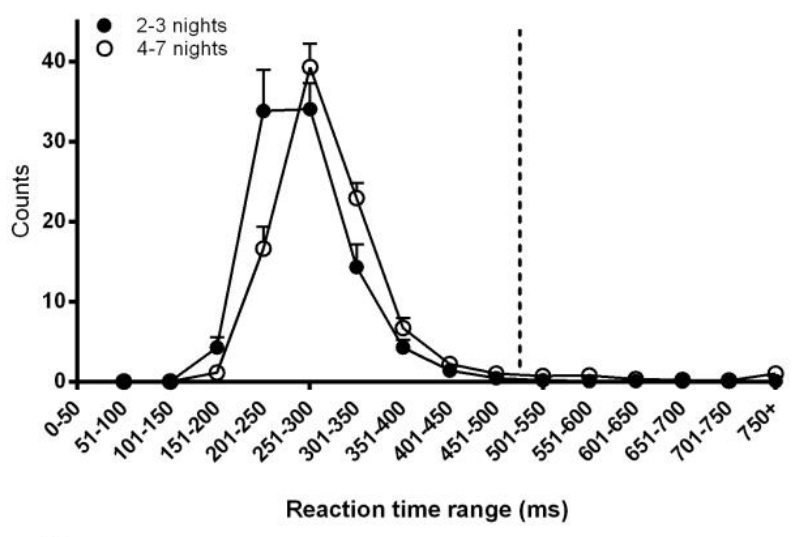

D

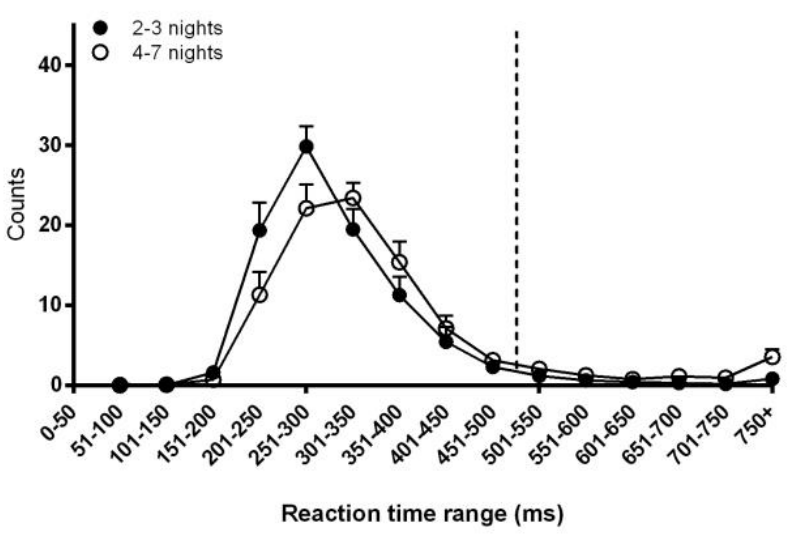

Figure 2. Comparison of total sum of responses occurring across the RT range for night shift workers who work either 2-3 or 4-7 consecutive nights at both the start of shift (B) and the end of shift (D). Values represent mean number of responses for a RT \pm standard deviation. Cumulative PVT RT for night shift workers both at the start $(A)$ and end $(C)$ of shift. Values represent mean RT percentile and standard deviation at $5 \%$ increments. The $y$-axis represents percentile values. A rightward shift in the RT percentile is indicative of overall cognitive slowing.

in psychomotor performance were seen at the start of shift between night 1 and night 4 of the shift sequence (39). Furthermore, a study investigating neurobehavioral performance of nurses working consecutive 12-hour shifts demonstrated no significant differences at the start of shift after 3 consecutive night shifts (40).

Cumulative PVT reaction time distribution analysis has been used in both laboratory and operational environments to examine sleepiness-related neurobehavioral impairments $(17,18)$. We observed a shift to the right in the entire RT distribution at both the start and end of the simulated night shift among participants who worked 4-7 night shifts, compared to those who had worked 2-3 shifts. This result further supports the notion that cognitive function, defined as decrements in information processing speed, is compromised not only at the end but also at the start of a night shift, after exposure to a long sequence of consecutive night shifts. These findings have implications for alertness management strategies for night shift workers, suggesting that interventions to improve alertness should be applied as early as possible in the night shift.

Night shift workers who work consecutive shifts experience acute and chronic sleep deficiency (41-43) due to misalignment between the circadian pacemaker and the sleep-wake cycle (44), sleep disorders $(45,46)$ and insufficient sleep opportunity (47). We assessed sleep parameters in the 24 hours and the seven days preceding the simulated night shift. There were no group differences in any of the sleep parameters (sleep efficiency, sleep onset latency, total bedtime, total sleep time), but the direction of differences were consistently such that those in the 4-7 shift group had lower sleep duration and poorer sleep quality than those in the 2-3 shift group. Importantly, both groups appeared to be at risk of chronic sleep deficiency, reporting relatively short sleep duration: mean 6.3 hours and 5.6 hours for the 2-3 and 4-7 night shift groups, respectively. 
Although total sleep time was not significantly different between groups, the difference in mean total sleep time ( 0.7 hours) accumulated over successive days to result in a difference between groups of 5.4 hours over seven days. Future studies should examine the potential adverse impact of this magnitude of sleep deficiency in a larger sample of night shift workers.

Misalignment between the circadian pacemaker and the sleep-wake schedule is associated with impaired alertness during wake time (48). Indeed deleterious effects of circadian misalignment on cognitive performance have been reported in night shift field studies $(14,49)$. Adjustment of the circadian pacemaker to the day sleep/night work schedule is a gradual process, and even after a number of successive night shifts, alignment may only partially occur (50-54). We recently reported from the same group of participants, that those who undertook a simulated night shift during their biological night (and who thus show little evidence of circadian adaptation to night shifts) showed impaired neurobehavioral performance compared to those who undertook the night shift outside of their biological night (19). This finding was consistent with previous reports that adjustment (both partial and full) of the circadian pacemaker to night shift work attenuates performance deficits particularly over successive night shifts $(36,50$, 51 ), and that reducing circadian misalignment during night shift work improves performance $(51,55)$. In the present study, we show that the number of consecutive nights worked did not on average affect the degree of circadian adjustment - there were no significant differences in the timing of the aMT6s rhythm (acrophase) among those who worked 4-7 versus 2-3 nights successively. The large inter-individual variability we observed in the degree of circadian phase adjustment in both groups of shift workers may be explained by individual differences in light-dark exposure, the sensitivity of the circadian pacemaker to light, and potentially exposure to non-photic time cues (56). Larger inter-individual differences were observed, particularly the timing of acrophase in the group exposed to 4-7 compared 2-3 night shifts. This may be attributed to individual differences in the direction and speed of circadian adaptation (12); however, it is difficult to conclude due to the limited sample size. Further work is required to determine the dynamics of circadian adaptation in a shift work population and furthermore, its relationship between neurobehavioral performance and the number of consecutive shifts worked.

Interestingly, this study found that at the end of the night shift, only mean RT was significantly lower among participants who worked 4-7 compared to 2-3 nights. A similar trend was observed for slowest $10 \%$ RT and number of lapses to increase in those who had worked 4-7 nights. It is unclear why the group differences in performance levels were more robust at the start of the night shift compared to the end of the night shift. It is possible that inter-individual differences in vulnerability to sleep deprivation (57) would be more prominent at the end of the night shift, when the duration of wakefulness is extended, thus giving rise to greater variation in RT. Another possible explanation for the lack of difference between groups at the end of the night shift is that there was a ceiling effect in performance impairment; perhaps both groups experienced equally high levels of sleepiness due to the combined effects of the circadian and homeostatic sleep drives during the latter part of the night shift.

Although this study contributes to our understanding of how features of the shift schedule (specifically, number of consecutive night shifts) impact upon alertness, there are some limitations. Firstly, although expected, total number of hours worked in the preceding 7 days was higher among the participants who worked 4-7 nights consecutively, in comparison to those working 2-3 nights. Therefore, differences in total number of hours worked may have contributed to the group differences we observed in alertness and performance. Ideally, a reference condition would be used (for example, day workers with comparable total number of hours worked) to account for this potential confound. We assessed neither the magnitude of impairment on the first night of a night shift sequence nor the dynamics of neurobehavioral impairment over successive night shifts within individuals. A previous study showed that the nocturnal decrease in subjective alertness was greatest on the first night shift compared with subsequent nights in the sequence (10). Individuals exposed to shift work may vary in their ability to tolerate and adapt to night shifts (51), which explains why some individuals experience shift work disorder (58). It is possible that the sample we studied was not representative of the night shift working population because of selection bias as the main study into which participants were recruited examined a light intervention to improve alertness in night shift workers. For example, a larger number of community/personal service workers were noted in individuals that work 2-3 consecutive night shifts. Additionally, a lack of information on shift history and type of shift schedules could contribute to this bias. Finally, although the sample size we used is comparable to previous studies with similar outcome measures (10, 18), we acknowledge that a larger sample size and/or a within subjects design may yield more robust findings.

We observed impaired performance at both the start and end of a simulated night shift among those who had worked between 4-7 compared to 2-3 consecutive nights. The observed attentional deficits, particularly relating to cognitive slowing, provide insights into the increased risk of occupational accidents and 
injuries as the number of consecutive shifts increase (59). For medical residents, the Institute of Medicine recommended that night float or night shift duty must not exceed four consecutive nights (60) due to the increasing risk of occupational accidents and injuries (61). Our findings, albeit in a non-medical working population provide evidence to support this recommendation by showing that performance is significantly impaired among individuals who have worked more than four consecutive nights, at both the start and end of night shift. Future studies should examine the impact of consecutive night shifts on more ecologically valid measures of occupational performance and error and the effectiveness of targeted alertness interventions for shift workers exposed to four or more consecutive night shifts, timed at the start and also the end of night shift.

\section{Acknowledgements}

The authors would like to thank Nicole Hennessy, Jade Murray, Dr. Suzanne Ftouni, research staff and students within the School of Psychological Sciences, Monash University and Darren O'Brien, Anna Mullins and Sue Wellington from Woolcock Institute of Medical Research for their assistance with data collection. We thank Mark Salkeld and the Adelaide Research Assay Facility at the University of Adelaide for performing the urine aMT6s radioimmunoassays. This research was supported by NHMRC project grant \# 545871.

\section{References}

1. Australian Bureau of Statistics. Working Time Arrangements. Australia, 2009 November. Report No.: 6342.0.

2. Kryger MH, Roth T, Dement WC. Principles and Practice of Sleep Medicine. 5th Edition ed. Philadelphia: Elsevier Saunders; 2010.

3. Knauth P, Landau K, Droge C, Schwitteck M, Widynski M, Rutenfranz J. Duration of sleep depending on the type of shift work. Int Arch Occup Environ Health. 1980;46(2):167-77. http://dx.doi.org/10.1007/BF00378195

4. Folkard S. Do permanent night workers show circadian adjustment? A review based on the endogenous melatonin rhythm. Chronobiol Int. 2008;25(2):215-24. http://dx.doi. org/10.1080/07420520802106835

5. Foret J, Benoit O. Sleep patterns of workers on rotating shifts. Electroencephalogr Clin Neurophysiol. 1974;37(4):377-44.

6. Akerstedt T. Shift work and disturbed sleep/wakefulness. Occup Med (Lond). 2003;53(2):89-94. http://dx.doi. org/10.1093/occmed/kqg046

7. Drake C, Gumenyuk V, Jefferson C, Roth T. Circadian Phase
Differences in Patients with Shift Work Disorder Relative to Asymptomatic Shift Workers. Sleep. 2011;34:A164-A.

8. Akerstedt T, Wright KP, Jr. Sleep Loss and Fatigue in Shift Work and Shift Work Disorder. Sleep Med Clin. 2009;4(2):25771. http://dx.doi.org/10.1016/j.jsmc.2009.03.001

9. Rogers AE, Hwang W-T, Scott LD, Aiken LH, Dinges DF. The Working Hours Of Hospital Staff Nurses And Patient Safety. Health Affairs. 2004;23(4):202-12. http://dx.doi.org/10.1377/ hlthaff.23.4.202

10. Santhi N, Horowitz TS, Duffy JF, Czeisler CA. Acute sleep deprivation and circadian misalignment associated with transition onto the first night of work impairs visual selective attention. PLoS One. 2007;2(11):e1233. http://dx.doi. org/10.1371/journal.pone. 0001233

11. Barnes RG, Forbes MJ, Arendt J. Shift type and season affect adaptation of the 6-sulphatoxymelatonin rhythm in offshore oil rig workers. Neurosci Lett. 1998;252(3):179-82. http://dx.doi. org/10.1016/S0304-3940(98)00585-0

12. Gibbs M, Hampton S, Morgan L, Arendt J. Adaptation of the circadian rhythm of 6-sulphatoxymelatonin to a shift schedule of seven nights followed by seven days in offshore oil installation workers. Neurosci Lett. 2002;325(2):91-4. http:// dx.doi.org/10.1016/S0304-3940(02)00247-1

13. Lamond N, Dorrian J, Roach GD, McCulloch K, Holmes AL, Burgess HJ, et al. The impact of a week of simulated night work on sleep, circadian phase, and performance. Occup Environ Med. 2003;60(11):e13. http://dx.doi.org/10.1136/ oem.60.11.e13

14. Ferguson SA, Kennaway DJ, Baker A, Lamond N, Dawson D. Sleep and circadian rhythms in mining operators: limited evidence of adaptation to night shifts. Appl Ergon. 2012;43(4):695-701. http://dx.doi.org/10.1016/j. apergo.2011.11.003

15. Folkard S, Lombardi DA, Tucker PT. Shiftwork: Safety, sleepiness and sleep. Ind Health. 2005;43(1):20-3. http:// dx.doi.org/10.2486/indhealth.43.20

16. Folkard S, Lombardi DA. Modeling the impact of the components of long work hours on injuries and "accidents". Am J Ind Med. 2006;49(11):953-63. http://dx.doi. org/10.1002/ajim.20307

17. Myerson J, Robertson S, Hale S. Aging and intraindividual variability in performance: analyses of response time distributions. J Exp Anal Behav. 2007;88(3):319-37. http:// dx.doi.org/10.1901/jeab.2007.88-319

18. Anderson C, Sullivan JP, Flynn-Evans EE, Cade BE, Czeisler CA, Lockley SW. Deterioration of Neurobehavioral Performance in Resident Physicians During Repeated Exposure to Extended Duration Work Shifts. Sleep. 2012;35(8):1137-46. http://dx.doi.org/10.5665/sleep.2004

19. Ftouni S, Sletten TL, Nicholas CL, Kennaway DJ, Lockley SW, Rajaratnam SM. Ocular Measures of Sleepiness Are Increased in Night Shift Workers Undergoing a Simulated Night Shift Near the Peak Time of the 6-Sulfatoxymelatonin Rhythm. J Clin Sleep Med. 2015;11(10):1131-41. http:// dx.doi.org/10.5664/jcsm.5086 
20. Netzer NC, Stoohs RA, Netzer CM, Clark K, Strohl KP. Using the Berlin Questionnaire to identify patients at risk for the sleep apnea syndrome. Ann Intern Med. 1999;131(7):485-91. http://dx.doi.org/10.7326/0003-4819-131-7-19991005000002

21. Brazier JE, Harper R, Jones NM, O'Cathain A, Thomas $\mathrm{KJ}$, Usherwood T, et al. Validating the SF-36 health survey questionnaire: new outcome measure for primary care. BMJ. 1992;305(6846):160-4. http://dx.doi.org/10.1136/ bmj.305.6846.160

22. Buysse DJ, Reynolds CF, 3rd, Monk TH, Berman SR, Kupfer DJ. The Pittsburgh Sleep Quality Index: a new instrument for psychiatric practice and research. Psychiatry Res. 1989;28(2):193-213. http://dx.doi.org/10.1016/01651781(89)90047-4

23. Johns MW. A new method for measuring daytime sleepiness: the Epworth sleepiness scale. Sleep. 1991;14(6):540-5.

24. Mastin DF, Bryson J, Corwyn R. Assessment of sleep hygiene using the Sleep Hygiene Index. J Behav Med. 2006;29(3):2237. http://dx.doi.org/10.1007/s10865-006-9047-6

25. Horne JA, Ostberg O. A self-assessment questionnaire to determine morningness-eveningness in human circadian rhythms. Int J Chronobiol. 1976;4(2):97-110.

26. Dinges DF, Pack F, Williams K, Gillen KA, Powell JW, Ott GE, et al. Cumulative sleepiness, mood disturbance, and psychomotor vigilance performance decrements during a week of sleep restricted to 4-5 hours per night. Sleep. 1997;20(4):267-77.

27. Van Dongen HP, Maislin G, Mullington JM, Dinges DF. The cumulative cost of additional wakefulness: dose-response effects on neurobehavioral functions and sleep physiology from chronic sleep restriction and total sleep deprivation. Sleep. 2003;26(2):117-26.

28. Jung CM, Ronda JM, Czeisler CA, Wright KP, Jr. Comparison of sustained attention assessed by auditory and visual psychomotor vigilance tasks prior to and during sleep deprivation. J Sleep Res. 2011;20(2):348-55. http://dx.doi. org/10.1111/j.1365-2869.2010.00877.x

29. Basner M, Dinges DF. Maximizing sensitivity of the psychomotor vigilance test (PVT) to sleep loss. Sleep. 2011;34(5):581-91.

30. Sinclair KL, Ponsford JL, Rajaratnam SM, Anderson C. Sustained attention following traumatic brain injury: use of the Psychomotor Vigilance Task. J Clin Exp Neuropsychol. 2013;35(2):210-24. http://dx.doi.org/10.1080/13803395.20 12.762340

31. Aldhous ME, Arendt J. Radioimmunoassay for 6-sulphatoxymelatonin in urine using an iodinated tracer. Ann Clin Biochem. 1988;25 (Pt 3):298-303. http://dx.doi. org/10.1177/000456328802500319

32. Dijk DJ, Duffy JF, Czeisler CA. Contribution of circadian physiology and sleep homeostasis to age-related changes in human sleep. Chronobiol Int. 2000;17(3):285-311. http:// dx.doi.org/10.1081/CBI-100101049

33. Nelson W, Tong YL, Lee JK, Halberg F. Methods for cosinor- rhythmometry. Chronobiol. 1979;6(4):305-23.

34. Barger LK, Sullivan JP, Vincent AS, Fiedler ER, McKenna LM, Flynn-Evans EE, et al. Learning to live on a Mars day: fatigue countermeasures during the Phoenix Mars Lander mission. Sleep. 2012;35(10):1423-35. http://dx.doi. org/10.5665/sleep. 2128

35. Lo JC, Groeger JA, Santhi N, Arbon EL, Lazar AS, Hasan $\mathrm{S}$, et al. Effects of partial and acute total sleep deprivation on performance across cognitive domains, individuals and circadian phase. PLoS One. 2012;7(9):e45987. http://dx.doi. org/10.1371/journal.pone.0045987

36. Lamond N, Dorrian J, Burgess H, Holmes A, Roach G, McCulloch K, et al. Adaptation of performance during a week of simulated night work. Ergonomics. 2004;47(2):154-65. http://dx.doi.org/10.1080/00140130310001617930

37. Waggoner LB, Grant DA, Van Dongen HP, Belenky G, Vila B. A combined field and laboratory design for assessing the impact of night shift work on police officer operational performance. Sleep. 2012;35(11):1575-7. http://dx.doi. org/10.5665/sleep. 2214

38. Rollinson DC, Rathlev NK, Moss M, Killiany R, Sassower $\mathrm{KC}$, Auerbach S, et al. The effects of consecutive night shifts on neuropsychological performance of interns in the emergency department: a pilot study. Annals Emergency Med. 2003;41(3):400-6. http://dx.doi.org/10.1067/mem.2003.77

39. Petru R, Wittmann M, Nowak D, Birkholz B, Angerer P. Effects of working permanent night shifts and two shifts on cognitive and psychomotor performance. Int Arch Occup Environ Health. 2005;78(2):109-16. http://dx.doi. org/10.1007/s00420-004-0585-3

40. Geiger-Brown J, Rogers VE, Trinkoff AM, Kane RL, Bausell $\mathrm{RB}$, Scharf SM. Sleep, sleepiness, fatigue, and performance of 12-hour-shift nurses. Chronobiol Int. 2012;29(2):211-9. http:// dx.doi.org/10.3109/07420528.2011.645752

41. Akerstedt T, Kecklund G, Knutsson A. Manifest sleepiness and the spectral content of the EEG during shift work. Sleep. 1991;14(3):221-5.

42. Tilley AJ, Wilkinson RT, Warren PS, Watson B, Drud M. The sleep and performance of shift workers. Hum Factors. 1982;24(6):629-41.

43. Lamond N, Dorrian J, Roach GD, Burgess HJ, Holmes AL, McCulloch K, et al. Performance, sleep and circadian phase during a week of simulated night work. J Hum Ergol (Tokyo). 2001;30(1-2):137-42.

44. Åkerstedt T. Shift work and disturbed sleep/wakefulness. Occup Med. 2003;53(2):89-94. http://dx.doi.org/10.1093/ occmed/kqg046

45. Sack RL, Auckley D, Auger RR, Carskadon MA, Wright KP, Jr., Vitiello MV, et al. Circadian rhythm sleep disorders: part I, basic principles, shift work and jet lag disorders. An American Academy of Sleep Medicine review. Sleep. 2007;30(11):146083.

46. Drake CL, Roehrs T, Richardson G, Walsh JK, Roth T. Shift work sleep disorder: prevalence and consequences beyond that of symptomatic day workers. Sleep. 2004;27(8):1453-62. 
47. Van Dongen HP, Maislin G, Mullington JM, Dinges DF. The cumulative cost of additional wakefulness: dose-response effects on neurobehavioral functions and sleep physiology from chronic sleep restriction and total sleep deprivation Sleep. 2003;26(2):117-29.

48. Wyatt JK, Ritz-De Cecco A, Czeisler CA, Dijk DJ. Circadian temperature and melatonin rhythms, sleep, and neurobehavioral function in humans living on a 20-h day. Am J Physiol. 1999;277(4 Pt 2):R1152-63.

49. Wright KP, Jr., Hull JT, Hughes RJ, Ronda JM, Czeisler CA. Sleep and wakefulness out of phase with internal biological time impairs learning in humans. J Cogn Neurosci. 2006;18(4):508-21. http://dx.doi.org/10.1162/ jocn.2006.18.4.508

50. Boudreau P, Dumont GA, Boivin DB. Circadian adaptation to night shift work influences sleep, performance, mood and the autonomic modulation of the heart. PLoS One. 2013;8(7):e70813. http://dx.doi.org/10.1371/journal. pone. 0070813

51. Crowley SJ, Lee C, Tseng CY, Fogg LF, Eastman CI. Complete or partial circadian re-entrainment improves performance, alertness, and mood during night-shift work. Sleep. 2004;27(6):1077-87.

52. Czeisler CA, Weitzman E, Moore-Ede MC, Zimmerman JC, Knauer RS. Human sleep: its duration and organization depend on its circadian phase. Science. 1980;210(4475):1264-7. http://dx.doi.org/10.1126/science.7434029

53. Dijk DJ, Duffy JF, Czeisler CA. Circadian and sleep/wake dependent aspects of subjective alertness and cognitive performance. J Sleep Res. 1992;1(2):112-7. http://dx.doi. org/10.1111/j.1365-2869.1992.tb00021.x

54. Smith MR, Fogg LF, Eastman CI. A compromise circadian phase position for permanent night work improves mood, fatigue, and performance. Sleep. 2009;32(11):1481-9.
55. Czeisler CA, Johnson MP, Duffy JF, Brown EN, Ronda JM, Kronauer RE. Exposure to bright light and darkness to treat physiologic maladaptation to night work. $\mathrm{N} \mathrm{Engl}$ J Med. 1990;322(18):1253-9. http://dx.doi.org/10.1056/ NEJM199005033221801

56. Arendt J. Shift work: coping with the biological clock. Occup Med. 2010;60(1):10-20. http://dx.doi.org/10.1093/occmed/ kqp162

57. Van Dongen HP, Baynard MD, Maislin G, Dinges DF. Systematic interindividual differences in neurobehavioral impairment from sleep loss: evidence of trait-like differential vulnerability. Sleep. 2004;27(3):423-33.

58. Gumenyuk V, Roth T, Drake CL. Circadian phase, sleepiness, and light exposure assessment in night workers with and without shift work disorder. Chronobiol Int. 2012;29(7):92836. http://dx.doi.org/10.3109/07420528.2012.699356

59. Barger L, Lockley S, Rajaratnam SW, Landrigan C. Neurobehavioral, health, and safety consequences associated with shift work in safety-sensitive professions. Curr Neurol Neurosci Rep. 2009;9(2):155-64. http://dx.doi.org/10.1007/ s11910-009-0024-7

60. Resident Duty Hours:Enhancing Sleep, Supervision, and Safety. Ulmer C, Wolman DM, Johns MME, editors. Washington, DC: The National Academies Press; 2009.

61. Lockley SW, Barger LK, Ayas NT, Rothschild JM, Czeisler CA, Landrigan CP, et al. Effects of Health Care Provider Work Hours and Sleep Deprivation on Safety and Performance. Joint Comm J Qual Patient Safety. 2007;33(11):7-18.

Received for publication: 9 December 2015 